\title{
Characterization of Mycobacterium chimaera in a heater-cooler unit in Latvia
}

\author{
Janis Kimsis $\mathrm{MSc}^{1}$, Ilva Pole MSc${ }^{2}$, Inga Norvaisa $\mathrm{MD}^{2}$, Uga Dumpis MD, $\mathrm{PhD}^{3}$ and Renate Ranka $\mathrm{PhD}^{1}$ (1) \\ ${ }^{1}$ Latvian Biomedical Research and Study Centre, Riga, Latvia, ${ }^{2}$ Riga East University Hospital, Riga, Latvia and ${ }^{3}$ Pauls Stradins Clinical University Hospital, \\ Riga, Latvia
}

To the Editor-Mycobacterium chimaera (M. chimaera) is an opportunistic environmental mycobacterium belonging to the $M$. avium-M. intracellulare complex. Transmission of $M$. chimaera from contaminated heater-cooler unit (HCU) water tanks to patients is a risk during open-heart surgery. ${ }^{1}$ Specifically, investigations have revealed that the contaminated HCU devices (Stockert 3T) came from one particular manufacturing facility, LivaNova in Munich, Germany, and were a likely source for cardiothoracic surgery-related severe $M$. chimaera infections that occurred in Switzerland, Germany, The Netherlands, and the United Kingdom. ${ }^{2}$ The importance of HCUs lies in their ability to regulate the body temperature of a patient during cardiac surgery. However, evidence suggests that the airborne transmission of aerosolized bacteria from the water tanks was responsible for these infections. ${ }^{2}$ Currently, the extent of global outbreaks is unknown, but the burden of invasive M. chimaera was estimated to be 156-282 cases per year in 10 countries where most cardiac-valve replacements are performed. ${ }^{3}$ We investigated the possible presence of $M$. chimaera in HCUs in the Cardiothoracic Department, Latvian Centre of Cardiology of Pauls Stradins Clinical University Hospital, Latvia. The water of HCUs was sampled before they underwent the routine disinfection procedure in May, 2017. Samples were cultured on Bactec MGIT 960 system (Becton Dickinson, Heidelberg, Germany). The growth of mycobacteria was observed in 2 samples. DNA was isolated and the $M$. chimaera was identified using a GenoType NTM-DR version 1.0 kit (Hain Lifescience, Nehren, Germany). Both isolates, designated LV-2017-1-HCU and LV-2017-2-HCU, were subjected to whole-genome sequencing with $150 \times$ coverage on an Ion Proton System (Thermo Fisher Scientific, Waltham, MA). For the bioinformatics analysis, reads in samples produced in this study were aligned to the $M$. chimaera reference genome JCM_14737 (ENA accession no. PRJNA324238) using the bwa MEM algorithm. Variants were called and marked based on the published criteria ${ }^{4}$ using BCFtools. Sequences of both isolates were compared to strains ZUERICH-1 (DSM 101591) and ZUERICH-2 (DSM 101592), representatives of the major M. chimaera groups 1 and $2 .^{5}$

For the phylogenetic analysis, raw sequencing reads of the isolates LV-2017-1-HCU and LV-2017-2-HCU were mapped against the M. chimaera type strain FI-01069 (DSM 44623) ${ }^{6}$ and compared with strain ZUERICH-1 and 127 other publicly available data sets of $M$. chimaera isolates belonging to subgroup 1.1 and previously collected in Denmark, Germany, The Netherlands, the United Kindgom, Ireland, the United States, Australia, and New Zealand (available in the European Nucleotide Archive; http://

Author for correspondence: Renate Ranka, E-mail: renate_r@biomed.lu.lv

Cite this article: Kimsis J, et al. (2021). Characterization of Mycobacterium chimaera in a heater-cooler unit in Latvia. Infection Control \& Hospital Epidemiology, 42: 1168-1170, https://doi.org/10.1017/ice.2020.1226 www.ebi.ac.uk/ena). ${ }^{4,5,7}$ The analysis was performed using CSI Phylogeny 1.4 software, Center of Genomic Epidemiology (https://cge.cbs.dtu.dk/services/CSIPhylogeny/), with default settings but including the reference in the final tree. ${ }^{8}$ Molecular phylogenetic analysis was performed using the maximum likelihood method. The phylogenetic tree was built from 176 SNP positions of $130 \mathrm{M}$. chimaera isolates and was visualized in MEGA6.

\section{Results}

In total, $2,555,838$ and $1,544,268$ sequencing reads were generated for samples LV-2017-1-HCU and LV-2017-2-HCU, respectively. The sequencing reads were deposited in the European Nucleotide Archive (ENA) under study numbers ERS3734298 and ERS37 34299.

Molecular phylogenetic analysis revealed that both Latvian HCU's samples were closely related to the strain ZUERICH-1 (Fig. 1A). Specific SNP signatures ${ }^{5}$ for subgroup 1.1. (substitutions of guanine (G) by adenine (A) at positions 113,518 and 209,278 of the DSM 44623T genome-GenBank accession no. LQOO00 000000) were found in both Latvian isolates. This result suggested that they belong to the subgroup 1.1 which contains most $M$. chimaera isolates from water systems or exhaust air of LivaNova HCUs in clinical use, isolates from HCUs sampled at the LivaNova production site, and isolates from related patients in different countries. ${ }^{5}$

During the phylogenetic analysis of the isolates LV-2017-1HCU and LV-2017-2-HCU, the sequencing coverage was $92.3 \%$ and $78 \%$ of the reference genome, respectively. The percentage of the reference genome covered by all isolates was $43.8 \%$. As expected, the isolates within the subgroup 1.1 showed little diversity, with a median pairwise distance of 2 SNPs (range, 0-40). ${ }^{5}$ A median pairwise distance for the Latvian LV-2017-1HCU and LV-2017-2-HCU isolates were 32 (range, 31-40) and 4 (range, 3-12) SNPs, respectively. The results showed that $M$. chimaera sequences from HCU in Latvia genetically clustered with the isolates from HCUs in The Netherlands, the United States, and Germany (samples 110, 128, 180, 187, 2015-22-15-01), as well as isolates from patients in The Netherlands (sample 198) and USA (samples 2015-22-63, 2015-22-79, 2015-22-80) (Fig. 1B).

\section{Discussion}

Overall, this is the first report of M. chimaera from HCUs in Latvia, which adds Latvia and the Baltic states to the global map of M. chimaera outbreaks associated with HCUs in hospitals. The presence of $M$. chimaera in the HCU in the university hospital in Latvia indicated the risk of exposure of patients that could lead to infection. Regular sampling of patients with chronic infections after cardiac surgery in Pauls Stradins Clinical University Hospital 
A
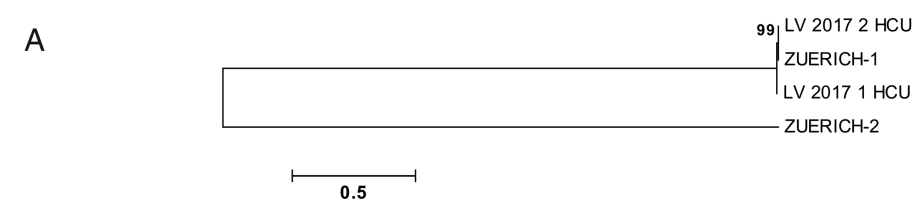

B

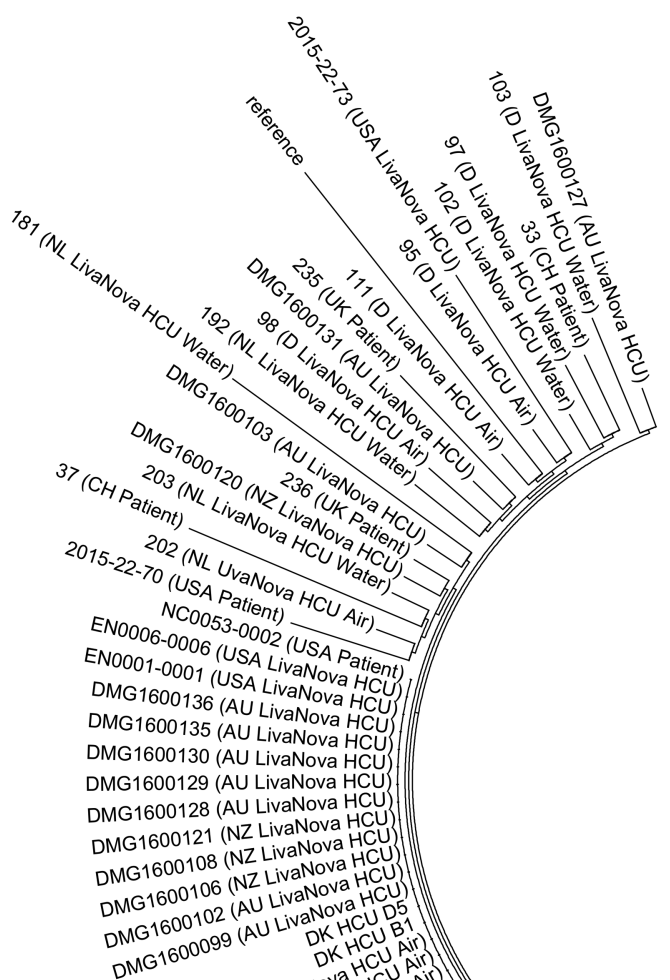

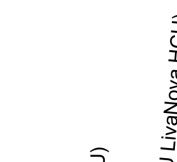

勇

定

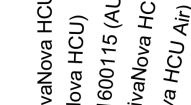

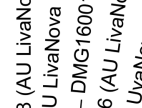

. 
Conflicts of interest. All authors report no conflicts of interest relevant to this article.

\section{References}

1. Sax H, Bloemberg G, Hasse B, et al. Prolonged outbreak of Mycobacterium chimaera infection after open-chest heart surgery. Clin Infect Dis 2015; 61:67-75.

2. Haller S, Höller C, Jacobshagen A, et al. Contamination during production of heater-cooler units by Mycobacterium chimaera potential cause for invasive cardiovascular infections: results of an outbreak investigation in Germany, April 2015 to February 2016. Euro Surveill 2016;21(17): pii=30215. doi: 10.2807/1560-7917.ES.2016.21.17.30215.

3. Sommerstein R, Hasse B, Marschall J, Sax H, Genoni M, Schlegel M, Widmer AF; Swiss Chimaera Taskforce. Global health estimate of invasive Mycobacterium chimaera infections associated with heater-cooler devices in cardiac surgery. Emerg Infect Dis 2018;24:576-578.

4. Svensson E, Jensen ET, Rasmussen EM, Folkvardsen DB, Norman A, Lillebaek T. Mycobacterium chimaera in heater-cooler units in Denmark related to isolates from the United States and United Kingdom. Emerg Infect Dis 2017;23:507-509.

5. van Ingen J, Kohl TA, Kranzer K, et al. Global outbreak of severe Mycobacterium chimaera disease after cardiac surgery: a molecular epidemiological study. Lancet Infect Dis 2017;17:1033-1041.

6. Tortoli E, Rindi L, Garcia MJ, et al. Proposal to elevate the genetic variant MAC-A, included in the Mycobacterium avium complex, to species rank as Mycobacterium chimaera sp. nov. Int J Syst Evol Microbiol 2004;54:1277-1285.

7. Williamson D, Howden B, Stinear T. Mycobacterium chimaera spread from heating and cooling units in heart surgery. N Engl J Med 2017;376:600-602.

8. Kaas RS, Leekitcharoenphon P, Aarestrup FM, Lund O. Solving the problem of comparing whole bacterial genomes across different sequencing platforms. PLoS One 2014;9(8):e104984.

9. Kasperbauer SH, Daley CL. Mycobacterium chimaera infections related to the heater-cooler unit outbreak: a guide to diagnosis and management. Clin Infect Dis 2019;68:1244-1250.

10. Kaelin MB, Kuster SP, Hasse B, et al. Schreiber PW. Diversity of nontuberculous mycobacteria in heater-cooler devices-results from prospective surveillance. J Hosp Infect 2020;S0195-6701(20)30105-5.

\title{
Impact of the pandemic on antimicrobial consumption patterns
}

\author{
Cristófer Farias da Silva PharmD ${ }^{1,2}$, Caroline Deutschendorf MD, MSc ${ }^{1}$, Fabiano Márcio Nagel MD ${ }^{1,2,3}$, \\ Camila Hubner Dalmora MD¹, Rodrigo Pires dos Santos MD, PhD ${ }^{1}$ and Thiago Costa Lisboa MD, PhD ${ }^{1,2,3}$ \\ ${ }^{1}$ Infection Control Committee, Hospital de Clínicas de Porto Alegre, Porto Alegre, Brazil, ${ }^{2}$ Programa de Pós Graduação em Ciências Pneumológicas/UFRGS, Porto \\ Alegre, Brazil and ${ }^{3}$ Department of Critical Care Medicine, Hospital de Clínicas de Porto Alegre, Porto Alegre, Brazil
}

To the Editor-Novel severe acute respiratory syndrome coronavirus 2 (SARS-CoV-2) impacts on economic, social, and healthcare systems. Uncertainties regarding coronavirus disease (COVID-19) promote concerns in choosing the best therapeutic strategy. Several drugs with antiviral effects were prescribed to treat COVID-19, but scientific evidence is not conclusive regarding benefit.

Unnecessary antimicrobial use may cause an increase in multidrug-resistant organisms. ${ }^{1,2}$ It is necessary to consider actions to prevent consequences that SARS-CoV-2 may have on antimicrobial use. ${ }^{1,2}$ Antibiotic stewardship is a strategy to promote the optimal use of antibiotics. SARS-CoV-2 probably changes the antibiotic consumption profile, and it is necessary to measure this difference.

Thus, our goal was to evaluate the impact of the pandemic on antimicrobial usage patterns comparing cohorts of SARSCoV-2-positive and SARS-CoV-2-negative patients admitted in specific hospital locations.

\section{Methods}

\section{Setting}

Hospital de Clínicas de Porto Alegre, a 845-bed university, tertiary-care, public hospital is located in the city of Porto Alegre, southern Brazil. On March 20, 2020, Brazil declared recognition of community-based coronavirus transmission across the country. It is the local reference for hospitalization of patients with

\footnotetext{
Author for correspondence: Thiago Costa Lisboa, E-mail: tlisboa@hcpa.edu.br Cite this article: da Silva CF, et al. (2021). Impact of the pandemic on antimicrobial consumption patterns. Infection Control \& Hospital Epidemiology, 42: 1170-1172, https://doi.org/10.1017/ice.2020.1227
}

suspected or confirmed COVID-19. At the pandemic moment, areas for COVID-19 isolation were created in the intensive care unit (ICU), the emergency department, and clinical wards.

\section{Study design}

A cross-sectional study was performed and data on antimicrobial consumption of May 2020 was included in our analysis. We adopted a "days of therapy" (DOT) methodology to measure antimicrobial consumption. ${ }^{3}$

\section{Data collection}

All hospital antimicrobial data from administrative databases were included, except antibiotics that are not audited by the infection control committee. We conducted an overall analysis and cluster analysis in COVID-19 and non-COVID-19 ICU, emergency department, and clinical ward. We selected the most used antimicrobial drugs in each cluster. Units were coupled per similarity to compare COVID-19 and non-COVID-19 antibiotics consumption.

\section{Statistical analysis}

We calculated antibiotics consumption based on DOT and adjusted per patient days (PD). We then compared this persontime rate with point estimates and confidence intervals for the incidence rate ratio considering Poisson distribution. The analysis was performed using Stata version 15.1 software (StataCorp, College Station, TX). 